\title{
Evaluating the Effects of Duloxetine on oxaliplatin Induced Peripheral Neuropathy Prophylaxis in Gastrointestinal Cancer Patients, randomized double-blind placebo controlled clinical Trial
}

rokhsareh soufi

shahid beheshti medical university pharmacy school

Maria Tavakoli Ardakani ( $D$ mariatavakoli@sbmu.ac.ir)

Shahid Beheshti University of Medical Sciences School of Pharmacy

shirin haghighi

Shahid Beheshti University of Medical Sciences School of Medicine

Research article

Keywords: oxaliplatin, duloxetine, chemotherapy induced peripheral neuropathy, prophylaxis

Posted Date: December 1st, 2020

DOl: https://doi.org/10.21203/rs.3.rs-115859/v1

License: (c) (i) This work is licensed under a Creative Commons Attribution 4.0 International License.

Read Full License 


\section{Abstract}

\section{Background}

oxaliplatin is a key drug in gastrointestinal (GI) cancer treatment. Peripheral Neuropathy is a troublesome and dose-dependent adverse effect of oxaliplatin. It can occur in two distinct forms: acute and chronic. Its incidence is estimated about 65-98 percent, of which 22 percent needed to stop chemotherapy. In some cases peripheral neuropathy has a long lasting impact on patient's quality of life. The purpose of this study is to evaluate the efficacy of duloxetine on the prevention of oxaliplatin induced peripheral neuropathy in $\mathrm{Gl}$ cancer patients.

\section{Methods}

In this randomized and double blind study, which was conducted in a tertiary teaching hospital, eligible patients were divided in to two groups. Treatment group was received Duloxetine the day before chemotherapy regimen initiation with the dose of $30 \mathrm{mg} /$ day for one week and then was titrated up to 60 $\mathrm{mg} /$ day until 12 weeks. One placebo capsule daily for one week then two capsules daily until 12 weeks was prescribed for placebo group. In every chemotherapy courses peripheral neuropathy was assessed by using NCl-CTCAE v4.03. Also chemotherapy related quality of life (QOL) at the baseline and 12 weeks was assessed by using FACT/GOG-NTX.

Results

Forty patients were randomized to the treatment and placebo group which were similar with chemotherapy regimen, type and stage of cancer. Data analysis of NCI-CTCAE showed duloxetine could prevent worsening of paresthesia more than placebo $(P=0.025)$ and patients in duloxetine group experience less peripheral sensory neuropathy $(P=0.001)$ than placebo. FACT/GOG-NTX analysis showed significant worsening of tingling and discomfort in hands (respectively $P=0.002,0.001$ ) and feet (respectively $P=0.017,0.019)$, having pain in hands and feet $(P=0.001)$ and difficulty breathing in cold temperature exposure $(P=0.023)$ in placebo compared with duloxetine group. In the other hand, duloxetine could not improve QOL $(P=0.06)$ and hadn't significant effects on Trouble feeling the shape of small objects in hand $(P=0.420)$ or Trouble buttoning buttons $(P=0.086)$.

\section{Conclusion}

Duloxetine can consider as a safe and effective medication for the prevention of oxaliplatin induced peripheral neuropathy in GI cancer. Nonetheless, more studies with larger population are needed.

Trial registration

IRCT20100127003210N15. Registered 25 Jun 2018, https://fa.irct.ir/user/trial/3256/view

\section{Background}


oxaliplatin is a third generation platinum compound which is considered as a key drug in gastrointestinal cancer treatment (1). One of the most common adverse effects of oxaliplatin is peripheral neuropathy which is abbreviated as oxaliplatin induced peripheral neuropathy (OIPN). Incidence of peripheral neuropathy due to oxaliplatin is high, according to one study the incidence rate of OIPN, when oxaliplatin was administrated $85-130 \mathrm{mg} / \mathrm{m}^{2}$, was $65-98 \%$ (2). It can occur in two distinct forms: acute and chronic. The most important trigger of acute peripheral neuropathy is cold temperature exposure. Chronic peripheral neuropathy risk factors are various such as: cumulative dose, time of infusion, history of chemotherapy induced peripheral neuropathy (CIPN) in previous courses and chemotherapy regimen (35). Chronic peripheral neuropathy is so hard to treat and in some cases it is not a reversible side effect (2). Peripheral neuropathy symptoms include paresthesia, tingling and numbness in hands and feet, pain in lower extremities and difficult breath in cold temperature exposure $(6,7)$. This adverse effect is so troublesome and has a negative impact on patient's Quality Of Life (QOL) (8). Duloxetine is a Serotonin, Norepinephrine Reuptake Inhibitor (SNRI drug) can impact on peripheral stimulants transmission because of its mechanism and also inhibits the activation of p38 phosphorylation, as a result, the activation and nuclear translocation of the NF-kB transcription factor is inhibited, reducing the inflammatory response and inhibiting nerve injury by regulating nerve growth factor $(9,14)$. These two neurotransmitters have a key role on suppression of peripheral stimulants transmission which can affect the sensation of pain (10). Duloxetine got FDA (Food and Drug Administration) approve for fibromyalgia, chronic muscle or joint pain and diabetic peripheral neuropathy treatment $(11,12)$. In most study Duloxetine showed positive effect on treating CIPN but based on our evaluation there is not any study that focus on duloxetine prophylactic effect on OIPN (1-4).

\section{Methods}

This randomized and double blind study was performed in Taleghani teaching hospital, Tehran, Iran. Patient's enrollment and follow up was started on July 2016 and finished in August 2018. This study was approved by Iranian Registry of Clinical Trials (IRCT20100127003210N15) and was confirmed by Ethics Committee of Shahid Beheshti University of Medical Sciences (IR.SBMU.PHNM.1395.418). Forty patients with Gastrointestinal cancer who are over 18 years old and assumed to receive first chemotherapy course based on oxaliplatin (new case patients who never expose to chemotherapy agents) were randomized (stratified by CIPN comorbid risk and chemotherapy regimen) to the treatment and placebo group.

Exclusion criteria include: uncontrolled hypertension (blood pressure more than 140/90 mm Hg), $\mathrm{ClCr}<30$ $\mathrm{ml} / \mathrm{min}$, liver impairment (ALT or AST > 3 Upper Normal Limit), liver metastasis, pregnancy,major drug interaction with Duloxetine and history of allergy to Duloxetine. We considered comorbid illness (eg diabetes mellitus) as a potential confounder so we assigned equal number of these patients to each group.

Treatment group received Duloxetine the day before chemotherapy regimen initiation with the dose of 30 $\mathrm{mg} /$ day for one week and then was titrated up to $60 \mathrm{mg} /$ day from second week until 12 weeks. One placebo capsule daily for one week then two capsules daily until 12 weeks was prescribed for placebo group. 
The primary outcome was the 12 weeks change in paresthesia and neuropathy severity which was assessed under supervision of a neurologist using NCI-CTCAE (every chemotherapy course) and FACT/GOG-NTX (at baseline and the end of the study which means twelfth week). In this questionnaire patients scored their neuropathy parameter (numbness, tingling, discomfort in hand and feet, difficulty hearing, joint pain and muscle cramp, buttoning buttons and having pain in extrimities and difficulty breathing in cold temperature exposure). Secondary outcome was 12 weeeks changes in CIPN related QOL which measured by FACT/GOG-NTX and FACT-C.

Due to the fact that these kind of parameters (paresthesia, numbness, pain and...) are subjective and strongly depend on the patient's pain tolerance threshold, so we decided to use an independent parameter named 12 weeks changes from baseline for determining the difference in patients neuropathy severity and QOL. Data analysis was done by IBM SPSS (version 24). Evaluation of data distribution was performed using the Shapiro-Wilk test. For analyzing categorical data, $\mathrm{Chi}^{2}$ test and for quantitative and independent data, Mann-Whitney was chosen. The $P$-value $\leq 0.05$ was considered to be significant.

According to table 1, there was no significant difference in gender, age, cancer type and stage, chemotherapy regimen and Para clinical parameters between 2 groups. At the baseline there was no significant difference in neuropathy condition between groups. The $P$-values for paresthesia $(0.603)$, peripheral sensory neuropathy (0.637) and peripheral motor neuropathy $(0.272)$ were more than 0.05 .

Table1. Baseline parameter in placebo and duloxetine group 


\begin{tabular}{|c|c|c|c|}
\hline Parameters & Placebo group & Drug group & P-value \\
\hline Mean Age & 59.6 & 55.75 & 0.545 \\
\hline Gender & & & 0.501 \\
\hline Female & 5 & 8 & \\
\hline Male & 15 & 12 & \\
\hline Cancer type & & & 0.642 \\
\hline Colon & 7 & 6 & \\
\hline Sigmoid & 4 & 4 & \\
\hline Gastric & 6 & 9 & \\
\hline Pancreas & 3 & 1 & \\
\hline Cancer stage & & & 0.756 \\
\hline Metastatic & 1 & 1 & \\
\hline Non-metastatic & 19 & 19 & \\
\hline Chemotherapy regimen & & & 0.513 \\
\hline Folfox ${ }^{1}$ & 18 & 18 & \\
\hline Xelox ${ }^{2}$ & 1 & 0 & \\
\hline Flot $^{3}$ & 1 & 2 & \\
\hline \multicolumn{4}{|l|}{ Para clinical } \\
\hline Albumin & 3.84 & 3.78 & 0.645 \\
\hline $\mathrm{Cr}$ & 1.04 & 1.06 & 0.222 \\
\hline Bili-total & 0.75 & 1.93 & 0.447 \\
\hline Bili-direct & 0.3 & 0.22 & 0.337 \\
\hline Medical history & & & 0.059 \\
\hline No medical history & 14 & 15 & \\
\hline DM & 3 & 0 & \\
\hline HTN & 0 & 3 & \\
\hline DM and HTN & 2 & 0 & \\
\hline Thyroid problems & 0 & 2 & \\
\hline Heart disease & 1 & 0 & \\
\hline
\end{tabular}


1. FOLFOX: folinic acid, fluorouracil and oxaliplatin ( $85 \mathrm{mg} / \mathrm{m}^{2}$ every 2 weeks)

2. XELOX: xeloda (capecitabine) and oxaliplatin ( $130 \mathrm{mg} / \mathrm{m}^{2}$ every 3 weeks)

3. FLOT: fluorouracil, leucovorin, oxaliplatin $\left(85 \mathrm{mg} / \mathrm{m}^{2}\right.$ every 2 weeks) and taxotere (docetaxel)

Patients were evaluated according to NCI-CTCAE questionnaire and response to intervention in our data analysis means patients have less or equal score at the end of the study compared with the baseline, patients in placebo group experience more paresthesia (the severity of paresthesia increase during 12 weeks from baseline) than treatment group (response to intervention: 19 patients in treatment group versus 13 patient in placebo group with P-value $=0.025)$. 12 weeks peripheral sensory neuropathy severity changes from baseline was more incremental in placebo versus treatment group (response to intervention: 19 patients in treatment group versus 10 patient in placebo group with P-value $=0.01$ ). There was not a significant changes from baseline in peripheral motor neuropathy (PMN) between 2 groups after 12 weeks (response to intervention: 19 patients in treatment group versus 17 patient in placebo group with $\mathrm{P}=0.107)$.

The results of FACT/GOG-NTX questionnaire for hand numbness (NTX1), feet numbness (NTX2), discomfort in hands (NTX3), discomfort in feet(NTX4), Joint pain or muscle cramps(NTX5), Trouble hearing (NTX6), Ringing in the ear (NTX7), Trouble buttoning buttons (NTX8), Trouble feeling the shape of small objects in hand (NTX9),Cold exposure induced lower extremities pain (NTX10),Cold exposure induced difficult breathing (NTX11) in both group have been showed in below table (table 3). These parameters were significantly different in NTX1, NTX2, NTX3, NTX4, NTX10, NTX11 between two group with better result in duloxetine group.

According to the results of FACT-C questionnaire, which include physical, emotional, social, functional and some other concerns, duloxetine couldn't improve the QOL.

Table2. Results of NCl-CTCAE

\begin{tabular}{|llll|}
\hline 12w change from baseline & response & No response & P-value \\
\hline Paresthesia & 13 & 7 & 0.025 \\
Placebo & 19 & 1 & \\
Treatment & & & 0.01 \\
PSN & 10 & 10 & \\
Placebo & 19 & 1 & 0.107 \\
Treatment & & & \\
PMN & 17 & 3 & \\
Placebo & 19 & 1 & \\
Treatment & & & \\
\hline
\end{tabular}


Page $7 / 14$ 


\begin{tabular}{|c|c|c|c|}
\hline FACT/GOG-NTX & response & No response & P-value \\
\hline Hands numbness (NTX1) & & & 0.002 \\
\hline placebo & 7 & 13 & \\
\hline treatment & 16 & 4 & \\
\hline Feet numbness (NTX2) & & & 0.017 \\
\hline Placebo & 12 & 8 & \\
\hline Treatment & 18 & 2 & \\
\hline Discomfort in hands (NTX3) & & & 0.001 \\
\hline Placebo & 8 & 12 & \\
\hline Treatment & 18 & 2 & \\
\hline Discomfort in feet (NTX4) & & & 0.019 \\
\hline Placebo & 12 & 8 & \\
\hline treatment & 17 & 3 & \\
\hline Joint pain or muscle cramps (NTX5) & & & 0.377 \\
\hline Placebo & 13 & 7 & \\
\hline treatment & 15 & 5 & \\
\hline Trouble hearing (NTX6) & & & 1.000 \\
\hline Placebo & 20 & 0 & \\
\hline Treatment & 19 & 1 & \\
\hline Ringing in the ear (NTX7) & & & 0.683 \\
\hline Placebo & 19 & 1 & \\
\hline Treatment & 19 & 1 & \\
\hline Trouble buttoning buttons (NTX8) & & & 0.086 \\
\hline Placebo & & 6 & \\
\hline \multirow[t]{2}{*}{ Treatment } & 14 & 3 & \\
\hline & 17 & & \\
\hline Trouble feeling the shape of small objects in hand (NTX9) & & & 0.420 \\
\hline \multicolumn{4}{|l|}{ Placebo } \\
\hline \multirow[t]{2}{*}{ Treatment } & 19 & 1 & \\
\hline & 18 & 2 & \\
\hline
\end{tabular}


Placebo

treatment

Cold exposure induced difficult breathing (NTX11)

Placebo

treatment

\section{Discussion}

Neuropathy is one of the bothersome side effects of chemotherapy which decrease patient's quality of life. This side effect is depended on doses and type of chemotherapy agent, frequency and duration of chemotherapy (5) This study is focus on OIPN prevention. At the end, results showed that prophylactic duloxetine can decrease the severity of the parameters such as paresthesia, peripheral sensory neuropathy, numbness and difficult breathing in exposure to cold temperature compared with placebo.

Yasuo Hirayama and et al, in 2015, demonstrated the effect of duloxetine on chemotherapy induced peripheral neuropathy treatment. In this study 34 patients who had received paclitaxel, oxaliplatin, vincristine, or bortezomib enrolled. The severity of pain and numbness was assessed using Visual Analog Scale (VAS). Although, we specifically focused on oxaliplatin and GI cancer patients and assessed our outcomes with NCl-CTCAE and FACT/GOG-NTX questionnaires, This article's results confirmed our study by showing better pain $(P$-value $=0.04)$ and numbness ( $P$-value $=0.03)$ relief in duloxetine group $(13)$.

In 2015, Akiko Otake and et al, performed a retrospectively review on medical records of 25 gynecological cancer patients treated with duloxetine for paclitaxel-induced peripheral neuropathy. At the end, results showed an improvement in 14 patient's neuropathy (11) although there are some differences between our RCT and their retrospectively review such as chemotherapy agent, patient's cancer type but their result like us confirm positive effect of duloxetine in decreasing peripheral neuropathy severity. One of the studies that are more similar to our RCT is Smith EM, et al clinical trial in 2013, with the aim of showing the efficacy of duloxetine in reducing the pain and treatment of chemotherapy induced peripheral neuropathy. Chemotherapy regimens consist of oxaliplatin, paclitaxel and other taxans and platinum regimen. As a primary outcome, patient's pain in duloxetine group decreased more than placebo ( $P$ value $=0.003$ ). Other finding like Quality of Life gets better results in duloxetine group (P-value $=0.01)$ (15).We worked on oxaliplatin induced neuropathy prevention and assessed more parameter such as paresthesia, sensory and motor neuropathy, cold induced numbness and difficult breathing but at the end both of us showed a significant decrease in painful neuropathy in duloxetine group. 
Ya-Hsu Yang and et al, conducted a clinical trial which is kind of resemble to ours.

Like our RCT, in this open labeled pilot study, 39 colorectal cancer patients that suffered from oxaliplatin induced peripheral neuropathy, participated and received duloxetine by increasing dose from $30 \mathrm{mg} / \mathrm{d}$ to $60 \mathrm{mg} / \mathrm{d}$. Pain and neuropathy severity was evaluated using VAS and NCI-CTCAE at the baseline and 12 weeks after duloxetine administration. According to their results duloxetine could improve neuropathy caused by oxaliplatin .similar to our study, adverse effects like dizziness, insomnia, nausea and etc. was reported during the study and because of them, 9 patients quitted the study (1) but in our study 5 patients in duloxetine group and 3 patients in placebo group leaved the study because of adverse effects.

The current clinical trial had strength and limitation. Strength include focus on prophylaxis of neuropathy, patients were receiving a specific regimen (taxan) and contains specific cancer type. Also there was some limitation in our study such as patient's lake of cooperation because long follow up duration.

\section{Conclusion}

Regarding to this double blind, placebo-treatment and randomized clinical trial, duloxetine could be effective on oxaliplatin induced peripheral neuropathy prophylaxis. Duloxetine was more effective on sensory neuropathy symptoms and it could decrease severity of hands neuropathy better than feet. It could give relief on cold exposure neuropathy like lower extremities paresthesia or difficult breathing.

\section{Abbreviations}

OIPN (Oxaliplatin Induced Peripheral Neuropathy), CIPN (Chemotherapy Induced Peripheral Neuropathy), QOL (Quality Of Life), SNRI (Serotonin Norepinephrine Reuptake Inhibitor)

\section{Declarations}

\section{Ethic and consent to participate}

This study was approved by Iranian Registry of Clinical Trials (IRCT20100127003210N15) and was confirmed by Ethics Committee of Shahid Beheshti University of Medical Sciences (IR.SBMU.PHNM.1395.418).All the patients were consented to participate in the study and signed the consent form. Clinical trial condition was explained to them.

Consent for publication: Not applicable

\section{Availability of Data and Materials}

All of the data analyzed during this study are included in this article.

\section{Competing interest}


The authors have no conflict of interest to declare.

\section{Funding}

The study was conducted with a grant provided by the shahid beheshti medical university, school of pharmacy.

\section{Acknowledgment}

This article is derived from the thesis Evaluating the Effects of Duloxetine on oxaliplatin Induced Peripheral Neuropathy Prophylaxis in Gastrointestinal Cancer Patients, a randomized double-blind placebo controlled clinical trial, supervised by Dr. Maria TavakoliArdakani and fulfillment of the requirement for the Degree of Pharm-D of Rokhsareh Soufi.

\section{Author's contribution}

Dr. TavakiliArdakani had full access to all the data in the study and take responsibility for the integrity of the data and the accuracy of the data analysis

\section{References}

1. Yang YH, Lin JK, Chen WS, Lin TC, Yang SH, Jiang JK, et al. Duloxetine improves oxaliplatininduced neuropathy in patients with colorectal cancer: an open-label pilot study. Supportive care in cancer : official journal of the Multinational Association of Supportive Care in Cancer. 2012;20(7):1491-7.

2. Argyriou AA, Polychronopoulos P, Iconomou G, Chroni E, Kalofonos HP. A review on oxaliplatininduced peripheral nerve damage. Cancer treatment reviews. 2008;34(4):368-77.

3. Grothey A. Clinical management of oxaliplatin-associated neurotoxicity. Clinical colorectal cancer. 2005;5 Suppl 1:S38-46.

4. Ocean AJ, Vahdat LT. Chemotherapy-induced peripheral neuropathy: pathogenesis and emerging therapies. Supportive care in cancer : official journal of the Multinational Association of Supportive Care in Cancer. 2004;12(9):619-25.

5. Quasthoff S, Hartung HP. Chemotherapy-induced peripheral neuropathy. Journal of neurology. 2002;249(1):9-17.

6. Stubblefield MD, Burstein HJ, Burton AW, Custodio CM, Deng GE, Ho M, et al. NCCN task force report: management of neuropathy in cancer. Journal of the National Comprehensive Cancer Network : JNCCN. 2009;7 Suppl 5:S1-S26; quiz S7-8.

7. Cavaletti G, Cornblath DR, Merkies IS, Postma TJ, Rossi E, Frigeni B, et al. The chemotherapyinduced peripheral neuropathy outcome measures standardization study: from consensus to the first validity 
and reliability findings. Annals of oncology : official journal of the European Society for Medical Oncology. 2013;24(2):454-62.

8. Mols F, Beijers T, Lemmens V, van den Hurk CJ, Vreugdenhil G, van de Poll-Franse LV. Chemotherapyinduced neuropathy and its association with quality of life among 2- to 11-year colorectal cancer survivors: results from the population-based PROFILES registry. Journal of clinical oncology : official journal of the American Society of Clinical Oncology. 2013;31(21):2699-707.

9. Berk M, du Plessis AD, Birkett M, Richardt D. An open-label study of duloxetine hydrochloride, a mixed serotonin and noradrenaline reuptake inhibitor, in patients with DSM-III-R major depressive disorder. Lilly Duloxetine Depression Study Group. International clinical psychopharmacology. 1997;12(3):13740 .

10. Willis WD, Westlund KN. Neuroanatomy of the pain system and of the pathways that modulate pain. Journal of clinical neurophysiology : official publication of the American Electroencephalographic Society. 1997;14(1):2-31.

11. Otake A, Yoshino K, Ueda Y, Sawada K, Mabuchi S, Kimura T, et al. Usefulness of duloxetine for Paclitaxel-induced peripheral neuropathy treatment in gynecological cancer patients. Anticancer research. 2015;35(1):359-63.

12. Aziz MT, Good BL, Lowe DK. Serotonin-norepinephrine reuptake inhibitors for the management of chemotherapy-induced peripheral neuropathy. The Annals of pharmacotherapy. 2014;48(5):626-32.

13. Hirayama Y, Ishitani K, Sato Y, lyama S, Takada K, Murase K, et al. Effect of duloxetine in Japanese patients with chemotherapy-induced peripheral neuropathy: a pilot randomized trial. International journal of clinical oncology. 2015;20(5):866-71.

14. Meng J, Zhang Q, Yang C, Xiao L, Xue Z, Zhu J. Duloxetine, a Balanced Serotonin-Norepinephrine Reuptake Inhibitor, Improves Painful Chemotherapy-Induced Peripheral Neuropathy by Inhibiting Activation of p38 MAPK and NF-kB. Frontiers in Pharmacology. 2019;10(365).

15. Smith EML, Pang H, Cirrincione C, Fleishman S, Paskett ED, Ahles T, et al. Effect of Duloxetine on Pain, Function, and Quality of Life Among Patients With Chemotherapy-Induced Painful Peripheral Neuropathy: A Randomized Clinical Trial. JAMA. 2013;309(13):1359-67.

\section{Figures}




\section{CONSORT 2010 Flow Diagram}

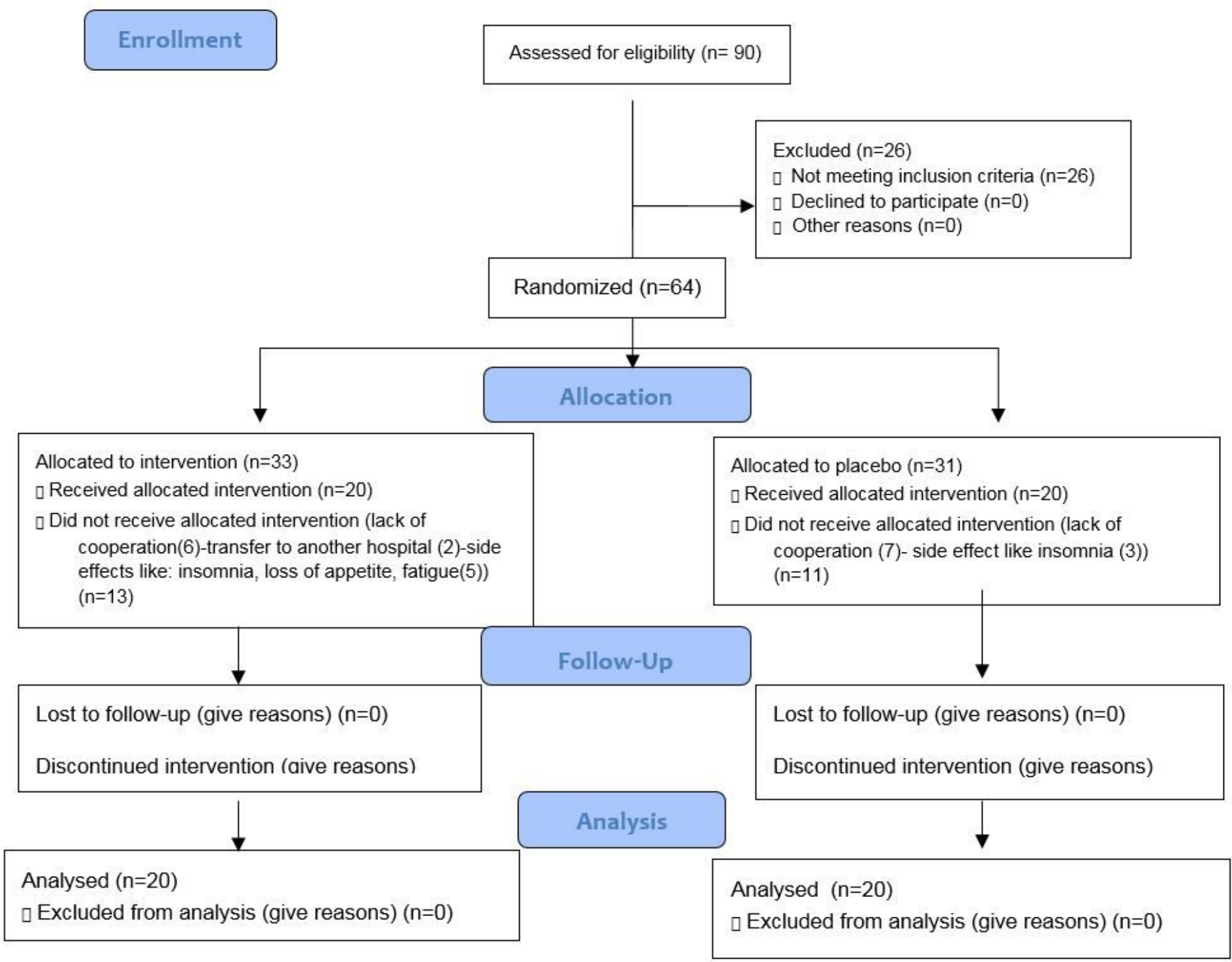

\section{Figure 1}

According to table 1, there was no significant difference in gender, age, cancer type and stage, chemotherapy regimen and Para clinical parameters between 2 groups. At the baseline there was no significant difference in neuropathy condition between groups. The P-values for paresthesia (0.603), peripheral sensory neuropathy $(0.637)$ and peripheral motor neuropathy $(0.272)$ were more than 0.05 . 

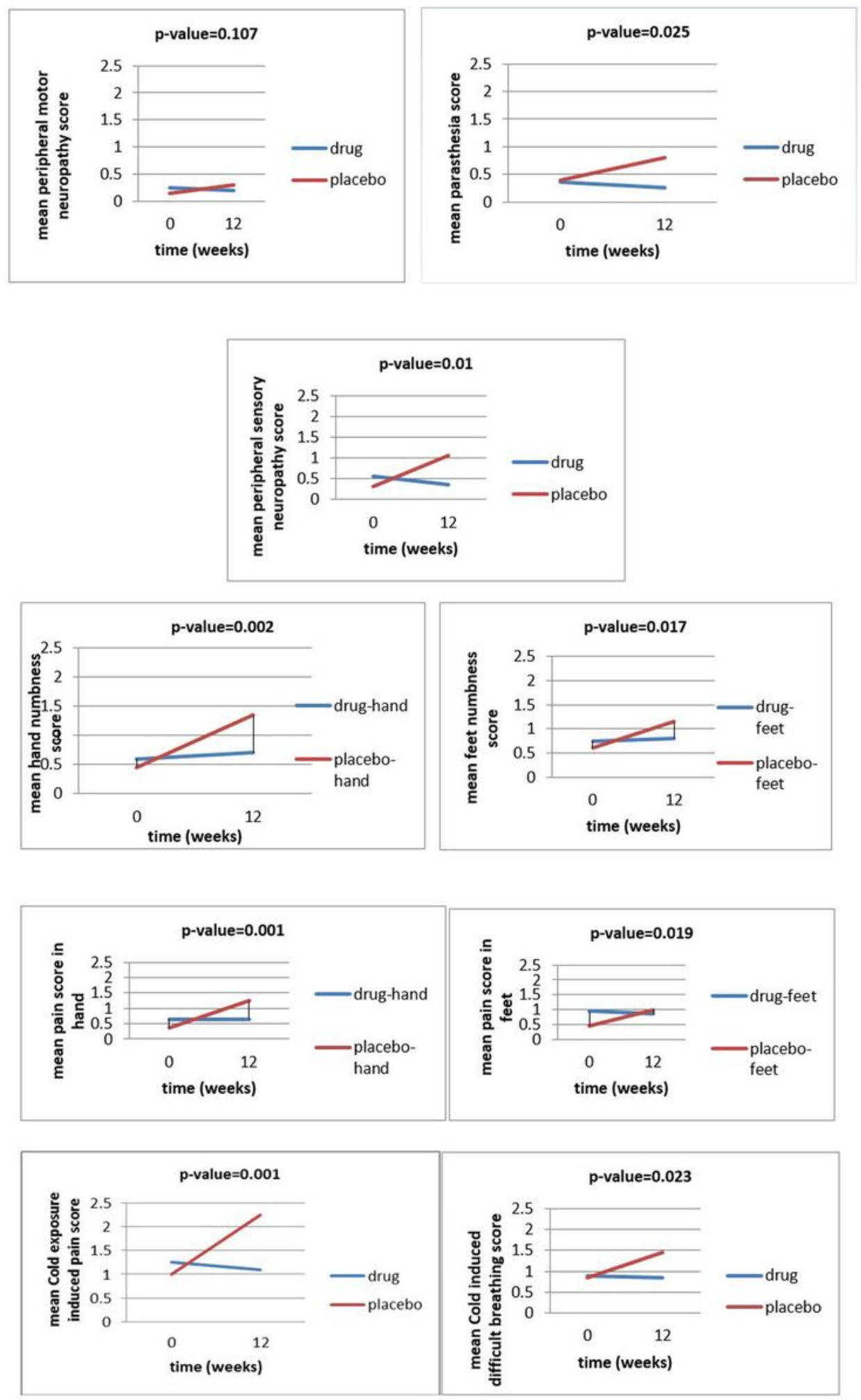

\section{Figure 2}

According to the results of FACT-C questionnaire, which include physical, emotional, social, functional and some other concerns, duloxetine couldn't improve the QOL 